\title{
Long-term Histologic Follow-up Study of Alcoholic Liver Disease
}

\author{
Yoshiharu Motoo, Toshinosuke WaKatsuki* and Yasuni NAKANUMA**
}

\begin{abstract}
Forty Japanese patients with alcoholic liver disease (nonspecific change, 9; fatty liver, 5; hepatic fibrosis, 4; chronic hepatitis, 12; alcoholic hepatitis, 5; liver cirrhosis, 5) were followed for 3-17 yr (average 8 yr) with repeated liver biopsies (2-5 times; average 2.5 times) at intervals of more than $3 \mathrm{yr}$. All of the patients continued to consume alcohol during this observation period. Five out of 12 patients with chronic hepatitis and 2 of 5 patients with alcoholic hepatitis eventually progressed to cirrhosis, while none of the 4 patients with hepatic fibrosis became cirrhotic. Anti-hepatitis $C$ virus antibody was positive in 2 patients with liver cirrhosis among 12 patients whose sera were available. Two patients with cirrhosis died of hepatic failure and one patient died of hepatocellular carcinoma. These data suggest that the long-term prognosis of alcoholic liver disease is not necessarily poor, but patients with chronic hepatitis or alcoholic hepatitis can be at risk of progression to cirrhosis.
\end{abstract}

(Internal Medicine 31: 33-38, 1992)

Key words: alcoholic hepatitis, fatty liver, hepatic fibrosis, chronic hepatitis, liver cirrhosis, repeated liver biopsies

\section{Introduction}

Although the amount and duration of alcohol intake and the progression of alcoholic liver disease are reported to be positively correlated (1), there are some reports which do not show a linear relationship between alcohol intake and the development of cirrhosis $(2,3)$. Several reports also suggest that immunological factors are involved in alcoholic liver disease $(4,5)$, and there seem to be some racial differences in the characteristics of alcoholic liver disease. For example, in Japan, patients with chronic hepatitis are often among alcoholic patients (6). Moreover, alcoholic fibrosis without features of alcoholic hepatitis is more commonly observed in Japanese heavy drinkers than in those of Western countries (7). Long-term follow-up of alcoholic liver disease seems necessary to clarify the long-term outcome of alcoholic liver disease of several histologic types among different geographic and socioeconomic conditions $(8-12)$. We have retrospectively analyzed the sequential liver biopsies and peritoneoscopic findings of 40 Japanese patients with alcoholic liver disease.

\section{Patients and Methods}

Patients who underwent two or more liver biopsies at intervals of more than $3 \mathrm{yr}$ between 1970 and 1989 were retrospectively analyzed. A total of 40 patients ( $35 \mathrm{men}$ and 5 women) were included in this study. The patients who had 2 biopsies at an interval of less than $3 \mathrm{yr}$ were excluded. Patients who had history of blood transfusion were also excluded. Liver biopsy was performed at approximately the seventh day after admission, and the frequency of biopsy was $2-5$ times per patient (average 2.5 times). The follow-up peroid was $3-17 \mathrm{yr}$ (average $8.3 \mathrm{yr})$. The age range of the patients was $26-70 \mathrm{yr}$ (average 48). Information on drinking habits was obtained from the patient records and cumulative alcohol consumption was calculated. The patients in this study habitually drank more than $80 \mathrm{~g} /$ day of ethanol for at least $5 \mathrm{yr}$.

Alcoholic liver disease was classified according to the criteria of Takeuchi et al (13). There are 6 different types of alcoholic liver disease in this criteria: alcoholic fatty liver, alcoholic hepatitis, alcoholic liver cirrhosis, alcoholic hepatic fibrosis, chronic hepatitis in habitual drinkers, and non-specific change. Hepatocellular injury, alcoholic

From Department of Internal Medicine, Cancer Research Institute, Kanazawa University, Kanazawa, * Department of Internal Medicine, Ushitsu General Hospital, Ushitsu and ** The Second Department of Pathology, Kanazawa University School of Medicine, Kanazawa Received for publication October 11, 1990; Accepted for publication May 13, 1991

Reprint requests should be addressed to Yoshiharu Motoo, MD, Department of Internal Medicine, Cancer Research Institute, Kanazawa University, 4-86 Yoneizumi, Kanazawa 921, Japan 


\section{Motoo et al}

hyaline (Mallory's bodies), polymorphonuclear infiltration in the lobules and portal areas, piecemeal necrosis, central and pericellullar fibrosis, giant mitochondria were the main features evaluated in this study. Hepatic fibrosis was classified as mild, moderate and severe as follows: mild, fibrosis hardly detected by hematoxylin and eosin (HE) stain but clearly found by silver stain; moderate, definite fibrosis shown by $\mathrm{HE}$ stain; and

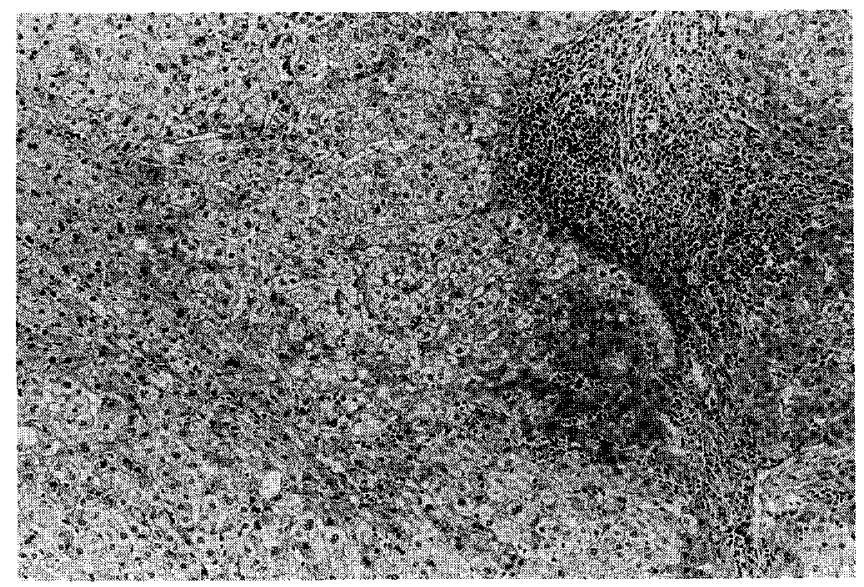

Fig. 1. Histology of chronic hepatitis in an alcoholic. Lymphocytic infiltration and lymph follicle formation in the portal area and piecemeal necrosis are seen $(H \& E$ stain, $\times 100)$.

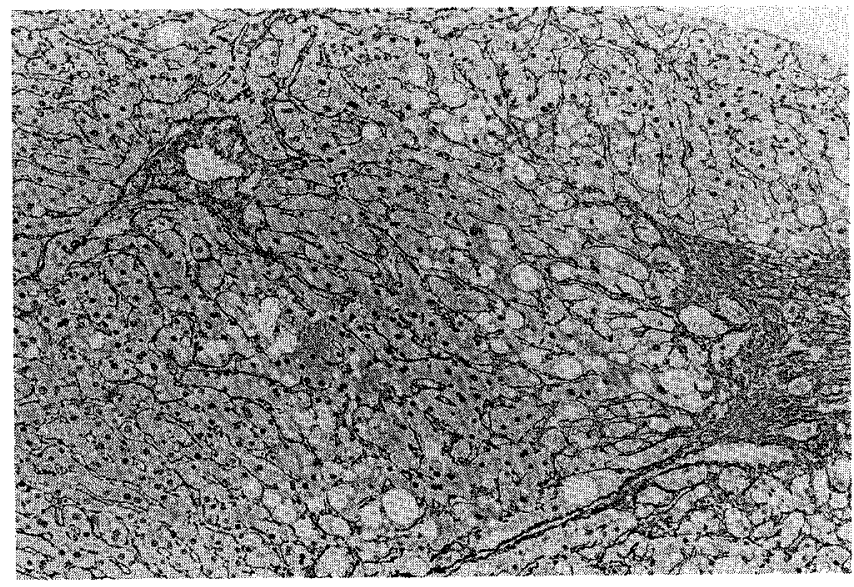

Fig. 2. Histology of alcoholic fibrosis shows moderate pericellular and periportal fibrosis and thickening of the terminal hepatic venule (reticulin stain, $\times 200$ ). severe, precirrhotic. The activity of chronic hepatitis was determined as in the case of viral hepatitis. The typical histology of chronic hepatitis (Fig. 1) and hepatic fibrosis (Fig. 2) is shown in this study.

Antibody to hepatitis $\mathrm{C}$ virus (anti-HCV) in sera of some patients was measured using an enzyme-linked immunosorbent assay (ELISA) kit (14) (Ortho HCV Ab ELISA test, Ortho Diagnostic Systems Inc., Raritan, NJ).

\section{Results}

The histologic diagnoses of the first liver biopsy are given in Table 1. All patients continued to drink during the observation period. Sequential histologic changes in each histologic category are described as follows (Table 2).

Non-specific change: Hepatic fibrosis developed in 7 out of 9 patients in this group. Two patients developed alcoholic hepatitis, and one showed features of severe fibrosis which were nearly compatible with the initial stage of cirrhosis. One patient in this group developed cirrhosis $7 \mathrm{yr}$ later, although biopsy was not done in the course of progression.

Fatty liver and hepatic fibrosis: Three of 5 patients with fatty liver showed the progression of fibrosis, and one patient with fatty liver developed cirrhosis $5 \mathrm{yr}$ later without any clinical features of alcoholic hepatitis. Perivenular fibrosis was seen in all patients with hepatic fibrosis in this study. Despite continuation of drinking, none of them developed cirrhosis.

Chronic hepatitis: Five out of 12 patients with chronic hepatitis progressed to cirrhosis. Four of these 5 patients showed the histologic features of chronic active hepatitis with or without lobular disorganization (LD) at least once in the course. Two of the 5 patients were female.

Alcoholic hepatitis: The main histologic characteristics were neutrophilic infiltration, ballooning of hepatocytes and central fibrosis. Mallory bodies were not recognized in patients with alcoholic hepatitis, nor with other types of alcoholic liver disease in this study. Two of 5 patients with alcoholic hepatitis progressed to cirrhosis, although no additional episodes of alcoholic hepatitis were found clinically. The period between the diagnosis of alcoholic

Table 1. Histologic Diagnosis at First Biopsy and Clinical Features $(\mathrm{M} \pm \mathrm{SD})$

\begin{tabular}{|c|c|c|c|c|c|}
\hline \multirow{2}{*}{ Histology } & \multirow{2}{*}{ Cases } & \multirow{2}{*}{$\begin{array}{l}\text { Age } \\
(\mathrm{yr})\end{array}$} & \multirow{2}{*}{$\frac{\operatorname{Sex}}{M / F}$} & \multicolumn{2}{|c|}{ Alcohol Intake (until first biopsy) } \\
\hline & & & & Duration (yr) & Total amounts $(\mathrm{kg})$ \\
\hline Nonspecific change (NC) & 9 & $45.9 \pm 7.4$ & $8 / 1$ & $22.0 \pm 9.5$ & $965.8 \pm 624.2$ \\
\hline Fatty liver $\quad(\mathrm{FL})$ & 5 & $53.8 \pm 4.7$ & $5 / 0$ & $25.0 \pm 7.7$ & $1,022.6 \pm 404.7$ \\
\hline Hepatic fibrosis & 4 & $47.5 \pm 4.5$ & $4 / 0$ & $23.8 \pm 4.1$ & $1,191.0 \pm 414.9$ \\
\hline Chronichepatitis $\quad(\mathrm{CH})$ & 12 & $49.5 \pm 8.7$ & $10 / 2$ & $25.4 \pm 9.5$ & $1,383.8 \pm 1,194.2$ \\
\hline Alcoholic hepatitis $(\mathrm{AH})$ & 5 & $44.8 \pm 14.2$ & $5 / 0$ & $22.4 \pm 13.3$ & $1,129.6 \pm 742.8$ \\
\hline Liver cirrhosis & 5 & $51.8 \pm 9.4$ & $3 / 2$ & $29.4 \pm 10.6$ & $1,594.4 \pm 670.0$ \\
\hline
\end{tabular}


Table 2. Histologic Course of Each Patient

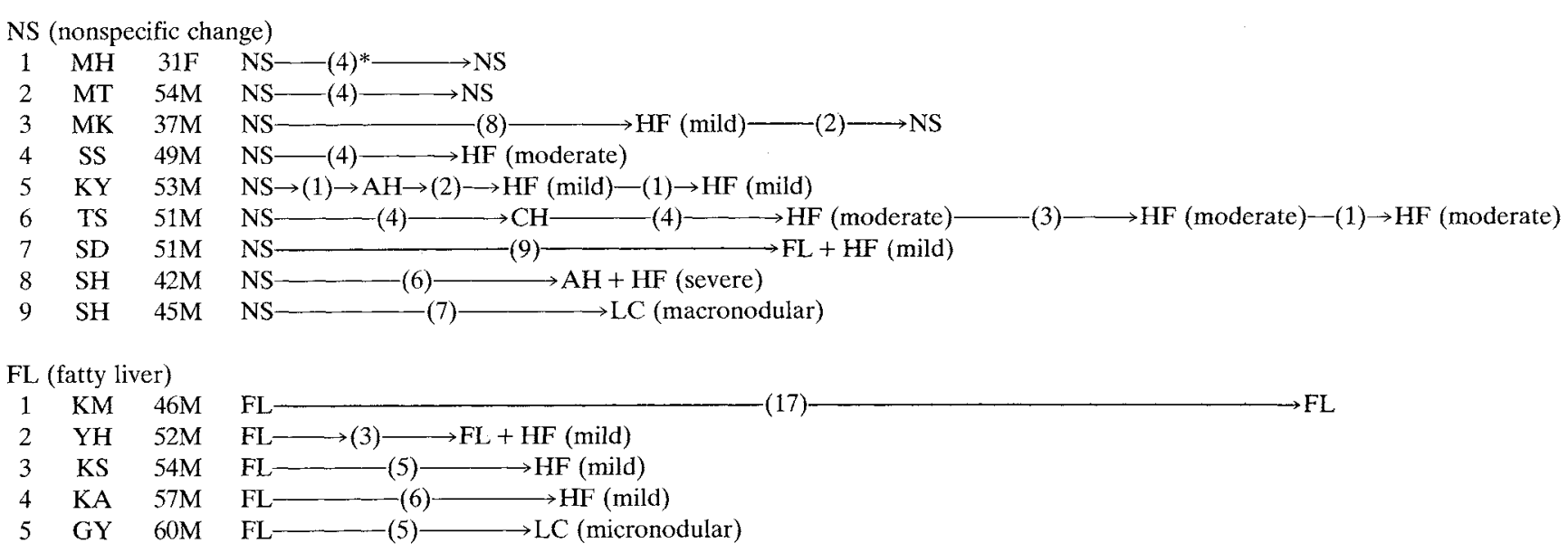

HF (hepatic fibrosis)

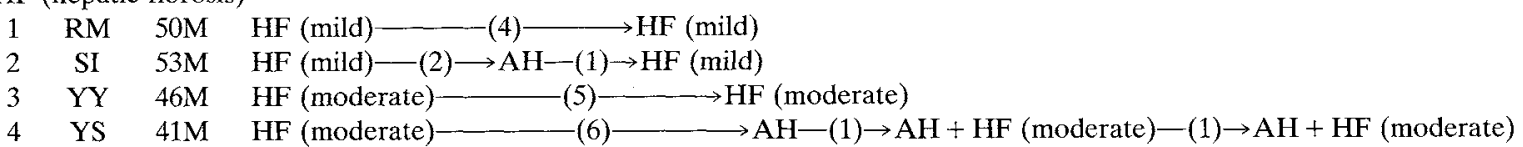

CH (chronic hepatitis)

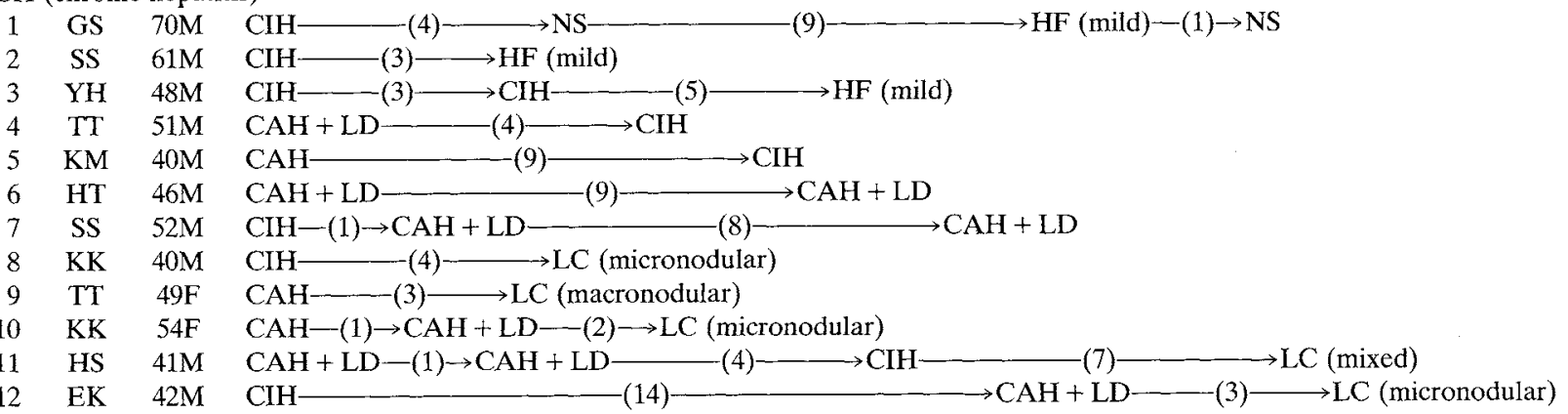

AH (alcoholic hepatitis)

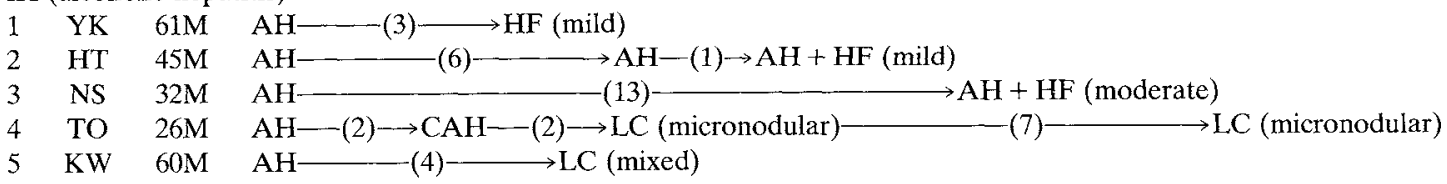

LC (liver cirrhosis)

$\begin{array}{lllll}1 & \text { OI } & 68 \mathrm{M} & \text { LC (macronodular) }-(3) \longrightarrow \text { hepatic failure (died) } \\ 2 & \text { MI } & 50 \mathrm{M} & \text { LC (micronodular) }-(3) \longrightarrow \text { hepatic failure (died) } \\ 3 & \text { EA } & 39 \mathrm{~F} & \text { LC (micronodular) } \\ 4 & \text { EU } & 53 \mathrm{M} & \text { LC (micronodular) } \longrightarrow \text { LC }+ \text { HCC (died) } \\ 5 & \text { TK } & 49 \mathrm{~F} & \text { LC (micronodular) } \longrightarrow \text { heart failure (died) }\end{array}$

* Parentheses indicate length of illness in yr.

hepatitis and that of liver cirrhosis was $4 \mathrm{yr}$ in both cases. One of the two patients who developed cirrhosis was histologically diagnosed as having chronic active hepatitis in the course of progression.

Liver cirrhosis: Four out of 5 patients with liver cirrhosis died. The causes of death were hepatic failure ( 2 cases), hepatocellular carcinoma ( 1 case), and heart failure (1 case).

Table 3 summarizes the sequential histological changes of the non-cirrhotic patients who progressed to cirrhosis. Seven out of 9 patients were initially diagnosed as having chronic hepatitis or alcoholic hepatitis. Female patients tended to develop cirrhosis more rapidly than male patients ( 3 yr vs $4-17 \mathrm{yr}$ ), despite a smaller cumulative alcohol intake volume (data not shown).

Anti-HCV was positive in 2 out of 12 patients whose sera were available for the serological test. One of the anti-HCV positive cases was LC at the first biopsy, and 
Table 3. Summary of the Histologic Course of Non-cirrhotic Patients who Progressed to Cirrhosis

\begin{tabular}{|c|c|c|c|c|}
\hline \multicolumn{2}{|c|}{ Case } & \multirow{2}{*}{$\frac{\text { Age }}{52}$} & \multirow{2}{*}{$\frac{\operatorname{Sex}}{\mathrm{M}}$} & Histologic course \\
\hline 1 & SH & & & NS $\longrightarrow(7)^{*} \longrightarrow$ LC (micronodular) \\
\hline 2 & GY & 65 & M & $\mathrm{FL} \longrightarrow(5) \longrightarrow \mathrm{LC}$ (micro) \\
\hline 3 & KW & 64 & M & $\mathrm{AH} \longrightarrow-(4) \longrightarrow \mathrm{LC}$ (mixed) \\
\hline 4 & TO & 37 & $\mathrm{M}$ & $\mathrm{AH}-(2) \rightarrow \mathrm{CAH}-(2) \rightarrow \mathrm{LC}($ micro $) \longrightarrow(7) \longrightarrow \mathrm{LC}($ micro $)$ \\
\hline 5 & KK & 44 & M & $\mathrm{CIH} \longrightarrow(4) \longrightarrow \mathrm{LC}$ (micro) \\
\hline 6 & EK & 59 & M & $\mathrm{CIH} \longrightarrow(14) \longrightarrow \mathrm{CAH}+\mathrm{LD} \longrightarrow-(3) \longrightarrow \mathrm{LC}$ (micro) \\
\hline 7 & $\mathrm{TT}$ & 52 & F & $\mathrm{CAH} \longrightarrow(3) \longrightarrow \mathrm{LC}$ (macronodular) \\
\hline 8 & KK & 57 & F & $\mathrm{CAH}-(1) \rightarrow \mathrm{CAH}+\mathrm{LD}-(2) \longrightarrow \mathrm{LC}($ micro $)$ \\
\hline 9 & HS & 53 & $\mathrm{M}$ & $\mathrm{CAH}+\mathrm{LD}-(1) \rightarrow \mathrm{CAH}+\mathrm{LD} \longrightarrow(4) \longrightarrow \mathrm{CIH} \longrightarrow-(7) \longrightarrow \mathrm{LC}$ (mixed) \\
\hline
\end{tabular}

* Parentheses indicate length of illness in yr.

Table 4. Measurement of Anti-HCV

\begin{tabular}{llllll}
\hline \multicolumn{2}{l}{ Patient } & & Histologic course* & Anti-HCV \\
\hline TK & 49 & F & LC $\rightarrow$ LC & + \\
EK & 42 & M & CH $\rightarrow$ LC & + \\
KW & 60 & M & AH $\rightarrow$ LC & - \\
NS & 32 & M & AH $\rightarrow$ HF & - \\
SS & 52 & M & CH $\rightarrow$ CH & - \\
YH & 48 & M & CH $\rightarrow$ HF & - \\
SS & 61 & M & CH $\rightarrow$ HF & - \\
YY & 46 & M & HF $\rightarrow$ HF & - \\
KA & 57 & M & FL $\rightarrow$ HF & - \\
TS & 51 & M & NS $\rightarrow$ HF & - \\
SD & 51 & M & NS $\rightarrow$ FL & - \\
MH & 31 & F & NS $\rightarrow$ NS & - \\
\hline
\end{tabular}

* Abbreviations as in Table 1.

the other had chronic hepatitis followed by development of liver cirrhosis. Anti-HCV negative cases included 2 patients with $\mathrm{AH}, 3$ patients with $\mathrm{CH}, 3$ patients with NS, 1 patient with HF and 1 patient with FL (Table 4).

\section{Discussion}

The present study showed the sequential histologic progression of several types of alcoholic liver disease in Japanese patients. The continuation of alcohol intake produced an increased incidence of hepatic fibrosis. Fibrotic change included perivenular fibrosis, but none of the patients with hepatic fibrosis developed cirrhosis. An observation period of 3 to $17 \mathrm{yr}$ (average: $8.3 \mathrm{yr}$ ) revealed that the progression rate to liver cirrhosis was not too high $(26 \%)$ and that the mortality of liver disease was low $(7.5 \%)$ in Japanese patients. Lelbach (1) reported a correlation between the death rate of cirrhosis and alcohol consumption in German patients. Nakano et al (15) showed that excessive alcohol intake led to fibrosis, especially perivenular fibrosis, which they reported to be a good indicator for cirrhosis in American patients. The differences between these studies and the present study might be due to racial and social factors. Alcohol metabolism is known to be dependent on genetic factors (16). In addition, the mode of habitual alcohol intake may considerably differ from country to country.

Ichinohe (9) reported the results of serial liver biopsies in 62 alcoholics in 1983. Histologic improvements were found in 25 patients who abstained from drinking. The remaining 37 patients continued drinking; 11 of 19 noncirrhotic patients progressed to cirrhosis. He emphasized the significance of portocentral (P-C) bridging fibrosis which was found in 8 of the 11 patients $(72.7 \%)$. In 9 of the 11 patients alcoholic hepatitis was histologically observed. Although the present study did not include any abstinent cases, the progression rate to cirrhosis in the present study was lower than that in Ichinohe's study $(25 \%$ vs $58 \%)$. No P-C bridging was seen in the patients of this study. The presence or absence of the P-C bridging might be the most important difference between his study and ours. Ichinohe's study did not include chronic hepatitis in alcoholics. He reported that 4 of 7 patients with fatty liver progressed to cirrhosis.

Ishida (10) also reported that in 40 patients who stopped drinking histological improvement was seen in $45.7 \%$ and that the histologically progressed cases had a high incidence of a history of blood transfusion, drug addiction or viral hepatitis. The cases with chronic active hepatitis developed cirrhosis at a high incidence, compared to those with chronic inactive hepatitis. Ishida's study seems to have included the patients with viral hepatitis. In the presnt study, patients who had a history of blood transfusion were excluded.

Habitual intake of alcohol is usually difficult to stop, and many patients continue to drink, although they decrease the amount of alcohol intake. For the patients in the present study no special treatment center for the care of severe alcohol addicts was necessary as in the study of Nakano et al (15). Moreover, chronic hepatitis induced by alcohol and hepatic fibrosis are the two common disease categories in alcoholic liver diseases in Japan. Therefore, our study may well reflect the characteristics of Japanese patients with alcoholic liver disease.

Although the number of hepatic fibrosis (HF) patients was small (4 cases) at the first biopsy in the present study, the number of patients with HF increased to 17 cases $(42.5 \%)$ including 6 cases with FL + HF or AH + 
$\mathrm{HF}$, whereas the number of patients with $\mathrm{CH}$ was 4 cases $(10 \%)$ at the final biopsy.

Perivenular fibrosis (PVF) has been reported to be a good indicator of progression to cirrhosis (15). In the present study PVF was seen in 12 patients. Three of the 12 patients progressed histologically (patient SD: NS $\rightarrow$ $\mathrm{FL}+\mathrm{HF}$ (mild), patient KS: FL $\rightarrow \mathrm{HF}$ (mild), and patient $\mathrm{KW}: \mathrm{AH} \rightarrow \mathrm{LC}$ ). PVF was not a good indicator of progression to cirrhosis, at least in this study. The severity of fibrosis at the initial biopsy might be involved.

Chronic hepatitis in alcoholics, which resembles viral hepatitis, is relatively common in Japan (6). In the present study, 12 out of 40 patients were initially diagnosed as having chronic hepatitis. Five of the 12 patients with chronic hepatitis progressed to cirrhosis in 3 to 17 (average 7.8) yr. Although it is difficult to eliminate the influence of non-A, non-B hepatitis virus (or hepatitis C virus) infection, which is presumed endemic in Japan, the improvement of the clinical and histological features by alcohol abstinence may support the alcoholic origin of the disease (6). The newly developed assay system for antibody to hepatitis C virus (14) may clarify the viral involvement in chronic hepatitis in alcoholics. We measured anti-HCV in 12 patients. Two patients with the final diagnosis of liver cirrhosis were anti-HCV positive. The involvement of $\mathrm{HCV}$ in the progression to cirrhosis might be considered in these 2 patients. Anti$\mathrm{HCV}$ is reported to be positive in $29.7-46.2 \%$ of alcoholic liver disease in Japan (17). In the present study the positive rate of anti-HCV was $17 \%$. Shimizu et al (18) presented the following anti-HCV positivity in various alcoholic liver diseases: $\mathrm{FL} 0 \%, \mathrm{AH} 0 \%, \mathrm{HF} 4.5 \%, \mathrm{CH}$ $73.3 \%$, and LC $46.2 \%$. Here, we could not assay anti$\mathrm{HCV}$ in all cases studied because stock sera of the earlier cases were not available, and some patients were not followed up at the time of study. Anti-HCV-negative patients with $\mathrm{CH}$ in our study showed the histologic features of HF later in the follow-up. The positivity of anti-HCV might depend on the time point of assay.

Women are reported to be more susceptible to alcoholrelated disease (19). In the present study, the female patients with chronic hepatitis progressed to cirrhosis more rapidly (within $3 \mathrm{yr}$ ) than male patients $(4-17 \mathrm{yr}$, average $7.5 \mathrm{yr}$ ), despite the smaller volume of cumulative alcohol intake. Since alcohol consumption has been increasing in Japan, it is likely that an increasing number of female patients with alcohol-related liver diseases will be found. Sequential biopsies will help to clarify the natural course of the disease in female patients.

Regarding alcoholic hepatitis, 2 out of 5 patients developed cirrhosis in $4 \mathrm{yr}$. Other cases showed fibrotic change in addition to the features of alcoholic hepatitis. Alcoholic hepatitis was seen in the course of other types of alcoholic liver diseases; 2 cases in the nonspecific change group, and 2 cases in the hepatic fibrosis group. However, these patients did not develop cirrhosis. The patients who were diagnosed as having alcoholic hepatitis at the first biopsy showed more severe features, both clinically and histologically, compared to the patients who were initially diagnosed as having nonspecific changes or hepatic fibrosis, and later developed alcoholic hepatitis. The progression to cirrhosis might be dependent upon the severity of alcoholic hepatitis.

In conclusion, chronic hepatitis is one of the common types of alcoholic liver disease in Japan, and the progression rate to cirrhosis is high in patients with chronic hepatitis. In this study no patients with hepatic fibrosis developed cirrhosis despite the continuation of alcohol consumption. Liver disease-related death occurred only in cirrhotic patients diagnosed at the first biopsy, and the long-term prognosis of the patients studied was not necessarily poor, but patients with chronic hepatitis or alcoholic hepatitis can be at risk of the development of cirrhosis.

Acknowledgments: We wish to thank Drs. N. Tanaka and R. Ohmizo for their cooperation.

\section{References}

1) Lelbach WK. Cirrhosis in the alcoholies and its relation to the volume of alcohol abuse. Ann NY Acad Sci 252: 85, 1975.

2) Brunt PW, Kew MC, Scheuer PJ, Sherlock S. Studies in alcoholic liver disease in Britain. 1. Clinical and pathological patterns related to natural history. Gut 15: 52, 1974.

3) Soerensen TIA, Orholm M, Bentsen KD, Hoeybye G, Eghoeje K, Christoffersen P. Prospective evaluation of alcohol abuse and alcoholic liver injury in men as predictors of development of cirrhosis. Lancet ii: 241, 1984.

4) Kakumu S, Lieber CS. Lymphocyte cytotoxicity in alcoholic hepatitis. Gastroenterology 72: 594, 1977.

5) Perperas A, Tsantoulas D, Portmann B, Eddeleston ALWF, Williams R. Autoimmunity to a liver membrane lipoprotein and liver damage in alcoholic liver disease. Gut 22: 149, 1981.

6) Nei J, Matsuda Y, Takada A. Chronic hepatitis induced by alcohol. Dig Dis Sci 28: 207, 1983.

7) Takada A, Nei J, Matsuda Y, Kanayama R. Clinicopathological study of alcoholic fibrosis. Am J Gastroenterol 77: 660, 1982.

8) Marbet UA, Bianchi L, Meury U, Stalder GA. Long-term histological evaluation of the natural history and prognostic factors of alcoholic liver disease. J Hepatol 4: 364, 1987

9) Ichinohe A. A follow-up study on the liver of alcoholics. Jpn J Gastroenterol 80: 2547, 1983 (in Japanese).

10) Ishida S. Long-term follow-up study of liver histology in alcoholic liver disease. Acta Hepatol Jpn 31: 402, 1990 (in Japanese).

11) Krasner N, Davis M, Portmann B, Williams R. Changing pattern of alcoholic liver disease in Great Britain: Relation to sex and signs of autoimmunity. Br Med J 1: 1497, 1977.

12) Takada A. Alcoholic liver disease: Its classification and pathogenesis. in: Biomedical and Social Aspects of Alcohol and Alcoholism, Kuriyama K, Takada A, Ishii M, Eds. Excerpta Medica, Amsterdam, 1988, p. 9.

13) Takeuchi J, Okudaira M, Takada A, et al. The incidence of alcoholic liver disease in Japan (1976-1985). Jpn J Gastroenterol 84: 1623, 1987 (in Japanese).

14) Kuo G, Choo Q-L, Alter $\mathrm{HJ}$, et al. An assay for circulating antibodies to a major etiologic virus of human non-A, non-B hepatitis. Science 244: 362, 1989.

15) Nakano M, Worner TM, Lieber CS. Perivenular fibrosis in 


\section{Motoo et al}

alcoholic liver injury: Ultrastructure and histologic progression. Gastroenterology 83: 777, 1982.

16) Goedde HW, Harada S, Agarwal DP. Racial differences in alcoholic sensitivity: A new hypothesis. Hum Genet 51: 331, 1979.

17) Yasuyama T. Anti-HCV in heavy drinkers. Jpn J Clin Med 49: 439, 1991 (in Japanese).
18) Shimizu S, Kiyosawa K, Sodeyama T, et al. Prevalence of antibody to hepatitis $\mathrm{C}$ virus in heavy drinkers with liver disease. Acta Hepatol Jpn 31: 589, 1990 (in Japanese).

19) Morgan MY, Sherlock S. Sex-related difference among 100 patients with alcoholic liver disease. Br Med J 1: 939, 1977. 